\title{
Investigation of MHD Impact on Argon Plasma Flows by Variation of Magnetic Flux Density
}

\author{
A. Knapp ${ }^{1}$, H. Fulge ${ }^{1}$, G. Herdrich ${ }^{*}, 1$, N. Ono ${ }^{2}$, R. Wernitz ${ }^{1}$, M. Auweter-Kurtz ${ }^{3}$, H.-P. Röser ${ }^{1}$ and \\ S. Fasoulas ${ }^{1}$ \\ ${ }^{1}$ Institute of Space Systems (IRS) - Universität Stuttgart, Stuttgart, Germany \\ ${ }^{2}$ Tohoku Gakuin University, Sendai, Japan \\ ${ }^{3}$ German Aerospace Academy (ASA), Stuttgart, 70569, Germany
}

\begin{abstract}
The interaction between a probe body and argon plasma flow is investigated to examine to what extent the probe head temperature and the bow shock distance can be influenced by applying a strong magnetic field. The experiments are performed using a strong permanent magnet installed inside a probe body with a spherical, coated probe head. Former investigations showed strong influence on the bow shock geometry but also on the inflow plasma jet. Several boundary conditions have been varied to evaluate their influence on the experiment. For an uncoated probe head the measured MHD impact was found to be of the same order of magnitude as for the coated case. Electrical isolation of the probe toward the vacuum chamber yielded only slight influence. The variation of the field strength was achieved by changing the amount of magnet segments installed. Pictures were analyzed to document the MHD interaction for each test case. It was found that the bow shock distance increased and the temperature of the probe head decreased while increasing the magnetic field density. This analysis precedes a thorough characterization of the plasma condition.
\end{abstract}

Keywords: Magnetohydrodynamics, plasma physics, Stuart number, plasma diagnostics

\section{INTRODUCTION}

Magneto-hydrodynamic (MHD) effects are present in many plasma processes used at the Institut für Raumfahrtsysteme [1]. Therefore, these MHD effects are studied on a basic research level. The current research focuses on the interaction of a strong neodymium permanent magnet, installed inside a probe body of the so called European standard, within an argon plasma flow. The expected MHD effects are to increase the bow shock distance and, as a consequence, possibly reduce the heat flux onto the probe body. These influences are currently analyzed experimentally and numerically by several research groups all over the world [2-8]. An excellent review of the work done within this field since the 1970s is given in references [6] and [8].

Earlier investigations using the thermal arcjet driven PWK4 at the Institute of Space Systems (IRS) yielded only weak interaction of the magnetic field and the argon flow due to the respectively low mass specific enthalpies, hence, the comparably lower electrical conductivities of the plasma conditions [8]. Thus, the whole setup has been revised and moved to a different plasma facility allowing the achievement of respectively higher mass specific enthalpies. The key parameters for this are the level of heat flux that can be achieved in combination with a respectively lower total pressure [9] as they can be provided for by the

*Address correspondence to this author at the Institute of Space Systems (IRS), University of Stuttgart, Pfaffenwaldring 29, Germany; Tel: +49 711 685 62412; Fax: +49 711685 63596; E-mail: Herdrich@ irs.uni-stuttgart.de magnetoplasmadynamically driven facility PWK1. Facilities as such are developed and in operation at IRS for the experimental investigation of aerothermodynamic effects. For this purpose, several plasma wind tunnels equipped with different types of plasma generators were built in order to cover the entire trajectory envelope of a space craft entering the atmosphere of a celestial body. In addition, basic investigations in the field of plasma radiation, material behavior and thermochemistry can be performed [1].

The basic concept of the recent MHD test campaign was to maximize the MHD impact to relevant properties such as heat flux, pressure and boundary layer. An indication for a possible MHD impact is the so called MHD interaction parameter, also known as Stuart number [10]

$$
S t=\frac{\sigma B^{2} L}{\left(1+\Omega_{e} \Omega_{i}\right) \rho_{\infty} v_{\infty}} .
$$

This parameter is defined as the ratio of the magnetic force (Lorentz force) to the inertia forces and is given e.g. by Macheret et al. [1], whereas the product of the electron and ion Hall parameter $\Omega_{\mathrm{e}} \Omega_{\mathrm{i}}$ is negligible within the examined argon plasma flows. The electrical conductivity of the plasma is represented by $\sigma, \mathrm{L}$ is the characteristic length of the MHD system. Here, $0.025 \mathrm{~m}$ was taken as this is the radius of the magnetic plasma probe used in this investigation. Flow parameters of the incoming flow are given through the density $\rho$ and the velocity $v$. It is commonly known that for $\mathrm{St}>1$ a definite MHD impact on the plasma flow can be observed. Analysis of existing and well-characterized MHD systems even show that with St in the order of magnitude of $10^{-1}$ impacts can already be noted 
[1]. As can be derived from this formula, the easiest way to increase the impact is to apply a stronger magnetic field. Disadvantageous is that the used permanent magnets reach their limit in terms of maximum magnetic flux density fairly early compared with coil based magnet designs.

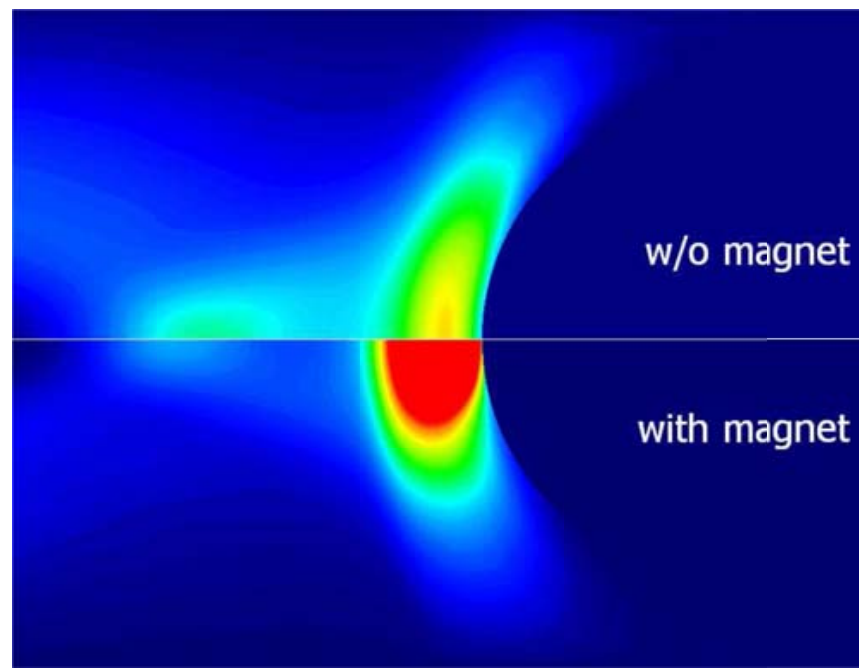

Fig. (1). False color images in comparison after the analyzing procedure.

Therefore, using the plasma facility PWK2 (derived from the German term "Plasmawindkanal" which stands for "plasma wind tunnel") together with the magnetoplasmadynamic (MPD) plasma source RD5, the ionization degree and thus the conductivity of the plasma flow was increased. In addition, the ambient pressure was also reduced by a factor of 4. The final plasma condition turned out to be a compromise in between maximizing the MHD effect on one side, but also keeping the system temperature below the operating temperature of the magnet.

Thus, reaching an ionization degree of about more than $30 \%$, the conductivity was increased by two orders of magnitude compared with the previously performed plasma conditions from the thermal arcjet RB3 driven PWK4. The bow shock is analyzed visually by taking photographs and comparing the geometries with and without magnetic interaction inside the boundary layer as shown in Fig. (1).

Furthermore, the center line intensities as well as the probe head temperatures are analyzed for each test case. In order to provide experimental data to support former proposed theories, several basic variations have been examined. These are the need of an isolating coating on the probe surface, the isolation of the probe towards the vacuum tank and the sensibility of the picture analysis mechanisms towards the evaluated color space.

In parallel, the test condition has also been analyzed numerically using the IRS SAMSA (Self and Applied field magnetoplasmadynamic thruster Simulation Algorithm) code, which has been originally designed to model the flow of applied field magnetoplasmadynamic (MPD) thrusters $[11,12]$. Due to the fact that SAMSA can also simulate the plasma generation itself, the experiment and numerical simulation have the same input variables. Furthermore, no arbitrary inflow conditions need to be generated given the plasma generation is modeled correctly.
The results of this experimental and numerical analysis will be compared, and discussed with respect to similar research activities [6, 13]

\section{EXPERIMENTAL SETUP}

The IRS plasma wind tunnel PWK1 is used for the performed investigations. More detailed information on this facility can be found in references [8] and [10].

\subsection{Test Facility and Plasma Source}

A $6 \mathrm{~m}$ long double-walled steel tank with a diameter of $2 \mathrm{~m}$ is used as cooled vacuum chamber; the plasma source is flanged from the outside to the conical part of the front lid.

This plasma wind tunnel facility (PWK1) is equipped with a 4-axis positioning system on which the different probes can be mounted. To achieve accessibility of the whole plasma free jet, adjustable optical windows are present on each side and on top of the tank. The plasma source used for the experiments is the MPD generator RD5, shown in Fig. (2). A detailed description of the vacuum chamber as well as the plasma source can be found in reference [4]. Operating conditions for the plasma generator used in these investigations can be found in Table $\mathbf{1}$.

\subsection{Probe Configuration}

The IRS probe body has a diameter of $50 \mathrm{~mm}$ which justifies the introduction of the radius for the characteristic length in equation (1). Due the overall diameter of $50 \mathrm{~mm}$ the probe belongs to the so-called European standard. However, the probe head itself is semi-spherical, see Fig. (3), which is in contrast to the standard blunt probe heads. Fig. (3) shows a schematic of the probe head interior whereas a photograph of both probe heads investigated is given in Fig. (4).

These heads are made of pure copper and were designed such that the surface of the neodymium permanent magnet rests $5 \mathrm{~mm}$ from the probe surface at the centerline. In case of the tests being performed with applied isolation towards the plasma flow, enamel was used as a coating on the probe head, whereas the rest of the probe body was coated with an isolating paint. High pressure cooling water at $20 \mathrm{bar}$ pressure is used to provide sufficient cooling of both probe and, in particular, the magnet. Two Pt100 thermometers, having $3 \mathrm{~mm}$ diameter each, were used as measuring equipment and installed in equally deep bore holes inside the probe head on the same reference circle diameter using heat conducting glue. The Kovar ${ }^{\circledR}$ insert is used to amplify the magnetic field strength in front of the probe in order to counteract the temperature based necessity to have the magnet stack shifted back from the stagnation point. Characteristics of the magnets are given in Table $\mathbf{2}$.

The maximum magnetic flux in front of the probe was measured to be $0.265 \mathrm{~T}$ with all 6 magnets present, whereas the maximum radial component was measured to be $0.12 \mathrm{~T}$ which results in an experiment specific Stuart number of about 30. This Stuart number is based on the maximum magnetic field strength at the stagnation point of the probe. Here, $\rho v$ was substituted by the mass flow rate divided by the cross-sectional area of the free jet at the measurement position. 


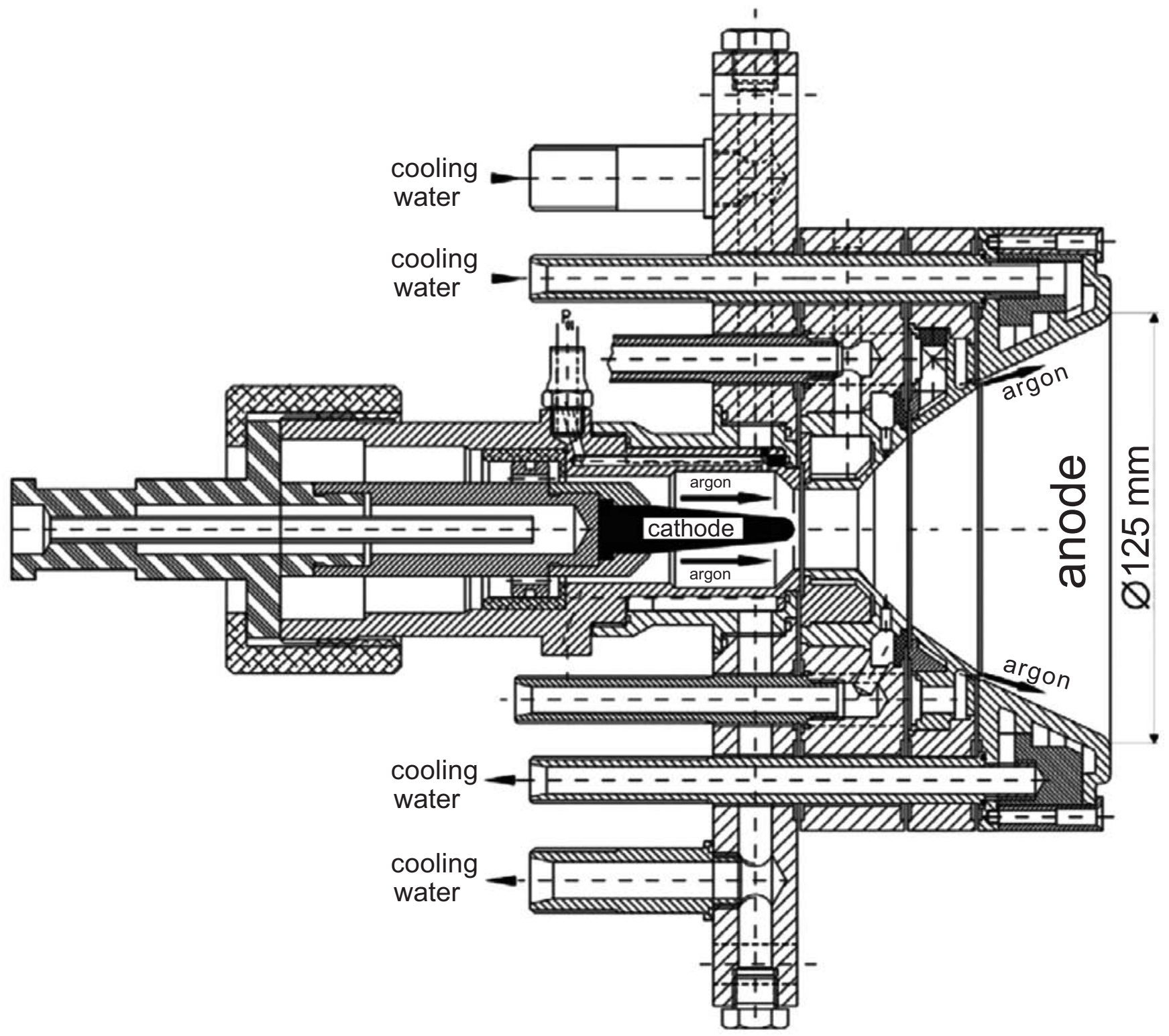

Fig. (2). MPD plasma generator RD5.

Table 1. RD5 Plasma Generator Conditions

\begin{tabular}{|c|c|c|c|}
\hline Mass Flow & Gas & Current & Ambient Pressure \\
\hline \hline $1,5+0,5 \mathrm{gs}^{-1}$ & Argon & $1040 \mathrm{~A}$ & $30 \mathrm{~Pa}$ \\
\hline
\end{tabular}

The symbols represent the measurement values, the lines are numerical simulations using Femm [14].

By varying the number of magnets installed it was possible to achieve a magnetic flux variation allowing a systematic investigation of the MHD effect. The experiments without magnet are performed using an identical but demagnetized neodymium rod in order to provide the system with the same structural properties (e.g. heat capacity).

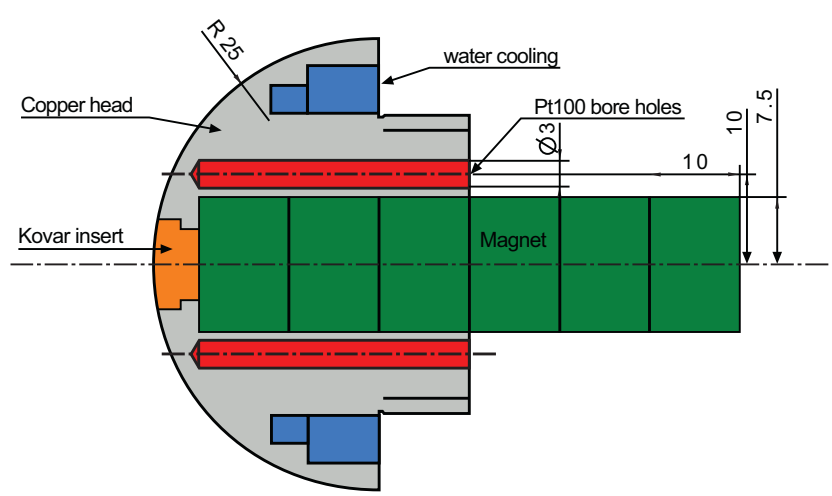

Fig. (3). Schematic of the used probe head. 


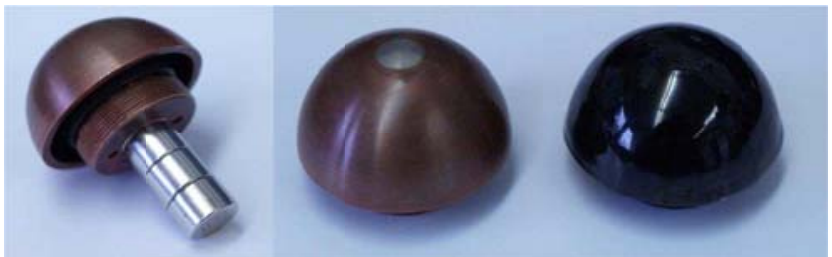

Fig. (4). Photograph of the used probe heads.

Table 2. Neodymium Permanent Magnet Characteristics

\begin{tabular}{|c|c|c|}
\hline Parameter & Value & Dimension \\
\hline \hline Diameter & 15 & $\mathrm{~mm}$ \\
\hline Length & 10 & $\mathrm{~mm}$ \\
\hline Material & $\mathrm{NeFeB}$ & - \\
\hline Material grade & $\mathrm{N} 35 \mathrm{EH}$ & - \\
\hline Remanence field strength & 1,25 & $\mathrm{~T}$ \\
\hline Max. operating temperature & 200 & ${ }^{\circ} \mathrm{C}$ \\
\hline Max. magnetic energy density & 295 & $\mathrm{~kJ} \mathrm{~m}^{-3}$ \\
\hline Coating & Nickel & - \\
\hline
\end{tabular}

Fig. (5) depicts the field strength distribution in front of the probe head.

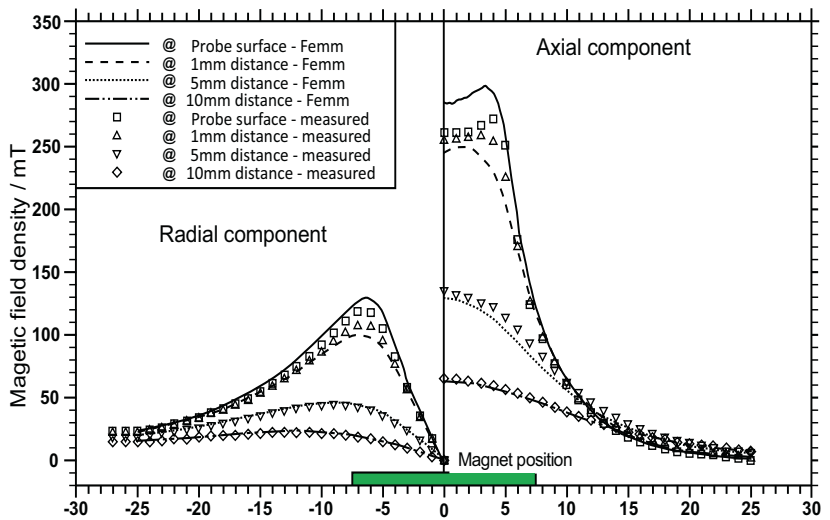

Fig. (5). Magnetic field distribution (6 magnets installed).

\subsection{MHD Setup}

The chosen plasma condition was characterized thoroughly using Pitot pressure, heat flux, enthalpy, Machcone and electrostatic triple probes [8].

For the analysis of the MHD impact on the bow shock geometry, a photographic setup was applied. In addition, emission spectroscopic measurements were performed in order to obtain valuable information about which argon lines contribute most to the observable plasma emission. Fig. (6) shows a schematic of the test setup. The probe body was placed at a distance of $130 \mathrm{~mm}$ from the nozzle exit, outside the flow. It was moved inside the plasma as soon as the plasma condition was stationary. The test duration was about 20 minutes.

The photographic setup was aligned in such a manner that the camera points perpendicularly towards the probe and was focused at the probe tip. Images of the plasma jet were recorded by a DSLR (Digital Single-Lens Reflex) camera (Olympus E-1, 5.1 mega pixels) through one of the viewing optical windows using an exposure time of $2 \mathrm{~ms}$. A newly

acquired lens permits a spatial resolution of the images of $0.075 \mathrm{~mm} /$ Pixel. The recorded image format is the OLYMPUS "RAW data format", which records signals directly from the CCD (charge-coupled device) in the camera. A raw data image is constructed out of a red, a blue and two green light intensities. This format is preferred due to the fact that no irreproducible camera specific image corrections like white balance, color saturation, contrast or image sharpness adjustment is used on the data. In the scope of this study, in contrast to Ono et al. [15], the blue intensity picture was utilized. The reason for this choice is on one hand the fact that the picture of the plasma appears blue as can be observed looking at Fig. (7). On the other hand, most of the argon ion lines (first ionization level) reside in the wavelength range between 300 and $550 \mathrm{~nm}$. In Fig. (8), a representatively measured emission spectrum is given with some identified lines. Using a magnet to achieve an MHD impact on the plasma, this impact is most likely to be observed for the ionized particles, ergo the blue part of the spectrum. The quantum efficiency of the E1 camera and the transmittance of the employed glass are shown in Fig. (9).

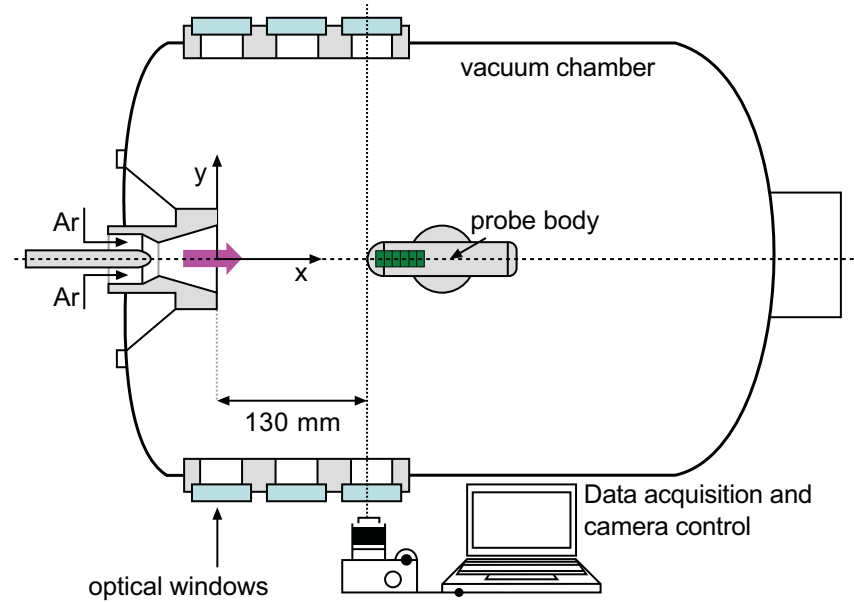

Fig. (6). Schematic view of the test setup.

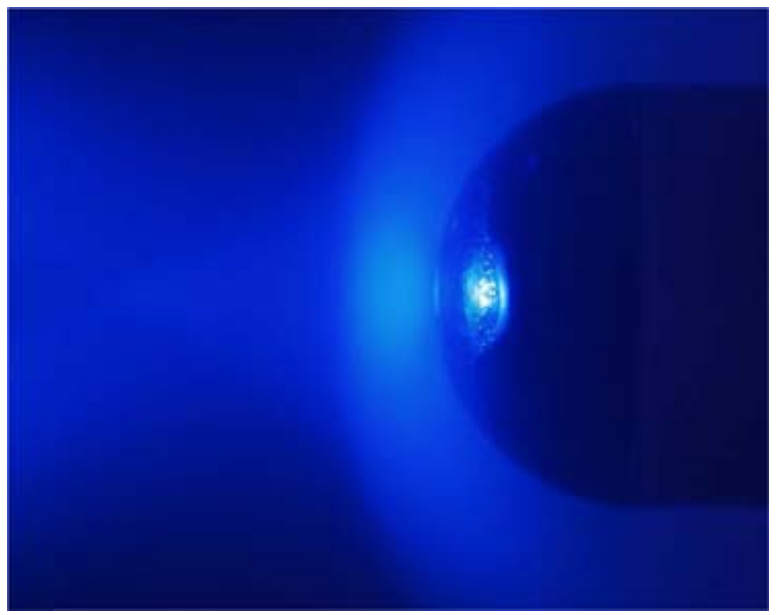

Fig. (7). Photograph of argon flow around probe body. 
The resolution of light intensities of the photographic pictures is $12 \mathrm{bit}$, or 4096 pixel. Table $\mathbf{3}$ depicts the parameters of the camera for the experiment condition.

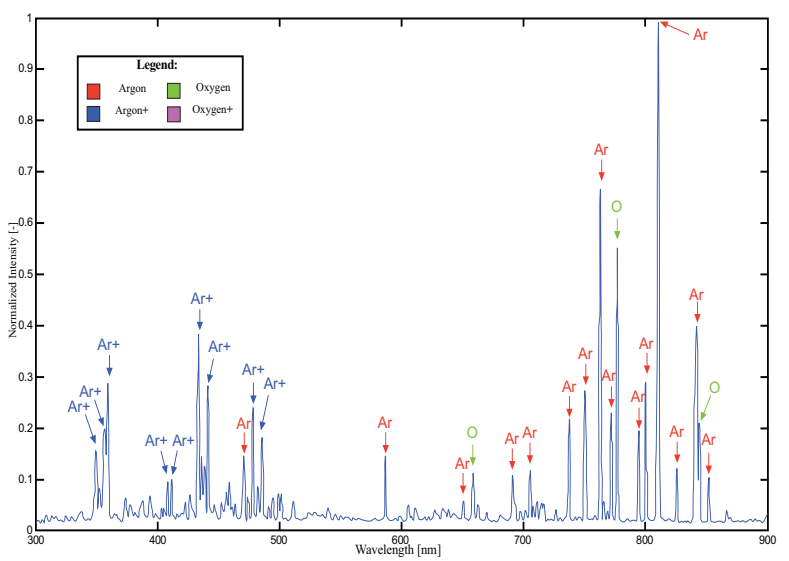

Fig. (8). Emission spectrum of experimental plasma condition.

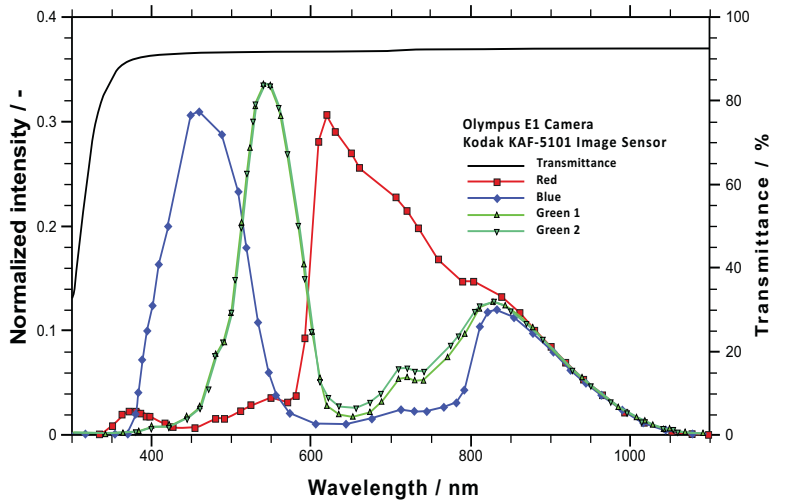

Fig. (9). Quantum efficiency and transmittance of the Olympus E1 Camera [16].

Image analysis software especially developed by Ono to study the plasma jet structure was applied to analyze the MHD effects that are accompanied by this plasma condition [15]. The camera was triggered via software and 12 image files were recorded and transferred directly to the hard drive of the computer. In order to account for actual MHD effects and to prevent misinterpreting momentary plasma fluctuations, all 12 pictures were added up and an average intensity value picture was generated. To analyze the bow shock distance, a procedure suggested in [17] has been taken under consideration. Here, the center line intensity is plotted against the distance from the probe tip and the shock is defined to be at position of the half maximum in front of the probe. A similar method was used in this work. The pictures were analyzed automatically using a MATLAB ${ }^{\mathrm{TM}}$ program. The bow shock position was defined to be located at the inflection point of the center line intensity profile. This was done in accordance to numerical studies at the IRS [18].

The whole procedure will be explained more closely in the Results section. Preceding this work, a thorough investigation of the plasma condition has been conducted. Table $\mathbf{4}$ grants an overview over the measured values of the most important characteristics.

Total pressure was measured using a Pitot probe, whereas the Mach number has been found to be better represented by cone probe measurement. A value for the heat flux was gained using a heat flux probe based on calorimetric measurement [19]. To get information about the local specific enthalpy a newly employed enthalpy probe, using mass injection principle was used [20]. Using an electrostatic triple probe, the electron temperature and the electron density were measured and the ionization degree as well as the electrical conductivity of the plasma was derived from these values. Electrostatic time of flight probes yielded no result for the flow velocity due to the fact that the plasma condition is too uniform. The given values for the velocity and the heavy particle temperature are estimations based on the already acquired measurements.

Table 3. Camera Characteristics

\begin{tabular}{|c|c|c|}
\hline Parameter & Value & Dimension \\
\hline \hline Shutter speed & $1 / 2000$ & $\mathrm{~s}$ \\
\hline ISO speed & ISO-200 & - \\
\hline Aperture & F/5.6 & - \\
\hline Focal length & 200 & $\mathrm{~mm}$ \\
\hline Measuring modus & spot & - \\
\hline Resolution of light intensities & 12 & $\mathrm{bit}$ \\
\hline
\end{tabular}

Table 4. RD5 Argon MHD Plasma Condition

\begin{tabular}{|c|c|c|}
\hline Parameter & Value & Dimension \\
\hline \hline X-Position & 130 & $\mathrm{~mm}$ \\
\hline Total pressure & $240 \pm 5$ & $\mathrm{~Pa}$ \\
\hline Heat Flux & $1263 \pm 108$ & $\mathrm{~kW} \mathrm{~m}^{-2}$ \\
\hline Mass specific enthalpy & $25,7 \pm 4,6$ & $\mathrm{MJ} \mathrm{kg}^{-1}$ \\
\hline Mach number & 2,1 & $\mathrm{na}$ \\
\hline Electron temperature & $17377 \pm 2604$ & $\mathrm{~K}$ \\
\hline Electron density & $7,38 \pm 0,1 \cdot 10^{19}$ & $\mathrm{~m}^{-3}$ \\
\hline Velocity & $\approx 3100$ & $\mathrm{~K}$ \\
\hline Heavy particle temperature & $\approx 8700$ & $\mathrm{~K}$ \\
\hline Ionization degree & 0.3 & - \\
\hline Electrical conductivity & 5660 & $\mathrm{~S} \mathrm{~m}^{-1}$ \\
\hline Experiment Stuart number & 30 & - \\
\hline
\end{tabular}

\section{NUMERICAL SIMULATIONS}

The original field of application for SAMSA is the numerical simulation of self - and applied field magnetoplasmadynamic (MPD) thrusters and generators [21]. It is based on a numerical code which has been developed and qualified at IRS for self-field MPD thrusters and which is currently enhanced to allow also for the simulation of applied-filed MPD thrusters [11].

First simulations showed that it is also capable of handling the plasma flow around a probe body as has been used for the current MHD experiment. However, one of the 
most interesting features of SAMSA is, that it can also simulate the effects inside the plasma generator and the input variables such as mass flow, ambient pressure, arc voltage, arc current and the magnetic field inside the probe body are therefore the same as for the experiment. Inside the code, the magnetic field is generated using a coil and a respective current to match the measured field strength of the permanent magnet.

Besides the feature of SAMSA to simulate the plasma generation process, it can also deal with the usual numerical approach using certain, defined inflow conditions. Thus, to simulate the plasma generation process is an asset to the program, not a fixed default. SAMSA provided already very good data in accordance to experimental results at the RB3 plasma source [15]. Based on this research and the characteristic of the code to easily implement different geometries of the plasma generation device, the RD5 generator was integrated and some results will be compared to the experimental data.

Fig. (10) shows the geometry data used for the current analysis. It also gives an example of the unstructured, adaptive grid used by the code.

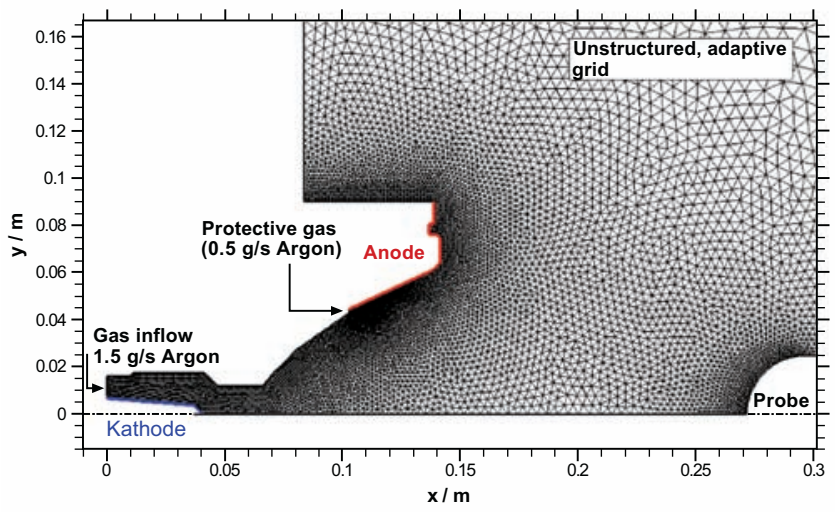

Fig. (10). Example for geometry input (RD5) for SAMSA and the grid used.

\section{RESULTS}

\subsection{Experimental Result}

Within the results, several pictures do not point out directly whether they were achieved using the coated or the bare probe head. If not marked differently, these pictures result from the reference case, which is always the fully isolated probe having the coated probe head installed.

\subsubsection{Temperature Measurements}

Pt100 resistance thermometers are located on the same reference circle diameter and both bores have the same depth, see Fig. (3). Nevertheless during all experiments they show different values up to a $\Delta \mathrm{T}$ of $2 \mathrm{~K}$. This discrepancy is most likely based on the Pt100s being not at the exact same depth inside the bore holes. Given the temperatures measured during the experiments of about $80{ }^{\circ} \mathrm{C}$, the discrepancy is still smaller than $10 \%$ and therefore acceptable.

Earlier research at the RB3 MHD condition showed no change in temperature for one of the Pt100 sensors, whereas the second sensor recorded an increase. This effect is now understood to have been the result of the inaccuracy of the thermometers. Taking this information into account, the temperatures did not change due to the magnet, thus the MHD interaction in the RB3 condition did not have any impact on the temperature. This in turn is understandable as the mass specific enthalpy for the RB3 condition is fairly low. Concluding the respective electrical conductivities in front of the probe are too low and the corresponding Stuart numbers do not imply an MHD effect.

Due to the fact, that the cooling water temperature is not constant for all experiments, a normalized reference temperature was defined in order to achieve comparable parameters. To reference the temperature all measured values were subtracted by the cooling water temperature of the inflow. This temperature difference was then divided again by the inflow cooling water temperature to become a normalized $\Delta \mathrm{T}$. The resulting formula is:

$$
\Delta T_{n o r m}=\frac{T_{P t 100-s e n s o r}-T_{c w ; \text { inflow }}}{T_{c w ; \text { inf low }}} .
$$

Following this procedure, the MHD impact on the temperature of the probe head has been measured. The temperature data has been recorded for several different test cases. As mentioned above one of the probe heads was coated, the other one was not. In addition, the influence of the probe body being isolated towards the vacuum chamber has been analyzed. The sensors have been named MHD1 and MHD2, the results are noted accordingly. The temperatures recorded within the uncoated case were altogether smaller. This is maybe due to the manufacturing tolerances of the bore holes. Thus, the measurement positions are not the same and the temperature levels are not comparable.

Fig. (11) depicts the results for the enamel coated probe head whereas Fig. (12) shows the same analysis for the bare, uncoated case.

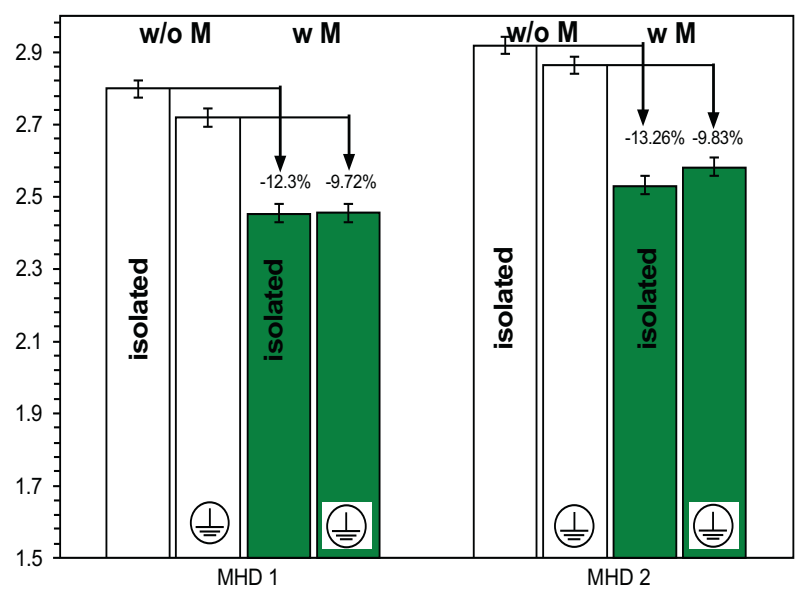

Fig. (11). Temperature data for the enamel coated probe head.

In case of applied coating on the probe head, both sensors measure a temperature reduction of about $10 \%$. The reduction in the isolated case seems to be slightly stronger which is balanced by the measurement inaccuracy increased due to the layer of the coating. Thus, the isolation was not found to have any effect on the temperature. 


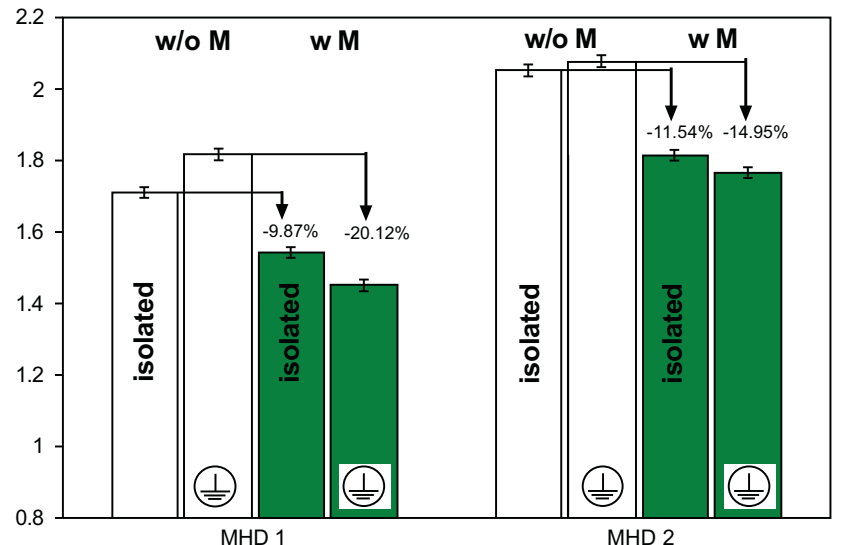

Fig. (12). Temperature data for the uncoated probe head.

In case of the uncoated probe head, both sensors also measure a temperature reduction in the order of $10 \%$. Here, the reduction in the earthed case seems to be slightly stronger which is again balanced by the measurement inaccuracy. Thus, the isolation was not found to have any effect on the temperature in this case as well.

Both diagrams represent the fact that due to an MHD impact, the temperature decreases. Isolating the probe from the tank does not affect the temperature of the probe head, keeping the inaccuracy of the measurement in mind. The interesting fact is that the temperature reduction is not only present in case of the blank probe head, but it is also in the same order of magnitude.

The isolated probe with an applied coating on the probe head is defined as the reference setup and is used to perform a variation of the magnetic field density hereafter. As described above, the field variation was achieved by installing different numbers of magnets inside the probe for the respective experiments. Table $\mathbf{5}$ gives the overall magnetic field strength at the probe tip with respect to the number of installed magnets.

Table 5. Magnetic Field Density at the Probe Tip with Respect to the Number of Magnets Installed

\begin{tabular}{|c|c|c|c|c|c|}
\hline $\begin{array}{c}\text { Number of Installed } \\
\text { Magnets }\end{array}$ & $\mathbf{1}$ & $\mathbf{2}$ & $\mathbf{3}$ & $\mathbf{4}$ & $\mathbf{6}$ \\
\hline \hline B in T (measured) & 0.200 & 0.225 & 0.243 & 0.254 & 0.265 \\
\hline B in T (Femm) & 0.202 & 0.241 & 0.253 & 0.258 & 0.262 \\
\hline
\end{tabular}

In the top part of Fig. (13), the change of $\Delta T_{\text {norm }}$ with respect to the number of magnets is shown. The lower part gives its reduction with respect to the magnetic field strength at the probe tip. Increasing the number of magnets leads to a reduction of the probe head temperature. This reduction seems to converge towards a constant value for more than 3 magnets, which is due to the fact that adding another magnet does not increase the magnetic field density at the tip much further. The slight increase for the last measurement is accounted for as measurement error.

A correlation between the measured temperatures and the heat flux onto the probe has been attempted. Using a 1D approach, the heat flux simply follows the temperature.
Thus, the temperature reduction of $10 \%$ of the stationary measurement equals a heat flux reduction of the same amount. Another analysis using ANSYS $^{\circledR}$ lead to the same result.
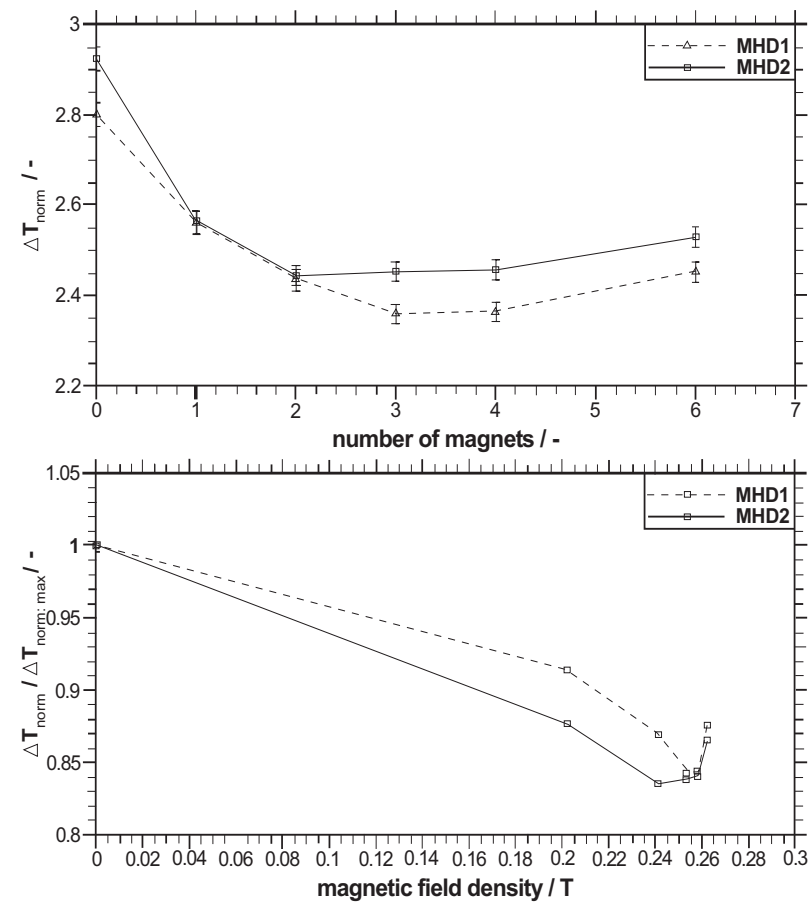

Fig. (13). Temperature change with respect to the number of magnets installed.

\subsubsection{Bow Shock Analysis}

The bow shock position was defined using the center line intensity profile and looking for the largest intensity gradient in front of the probe. Due to the fact that the coating reflected the illumination coming from the plasma generator, it had to be blackened in order to have a defined probe tip. The intensity value at the position of the maximum gradient was also set to zero to simplify the analysis. This was done for all pixel lines up to the probe diameter. Fig. (14) shows the averaged picture before and after the automatic analysis.

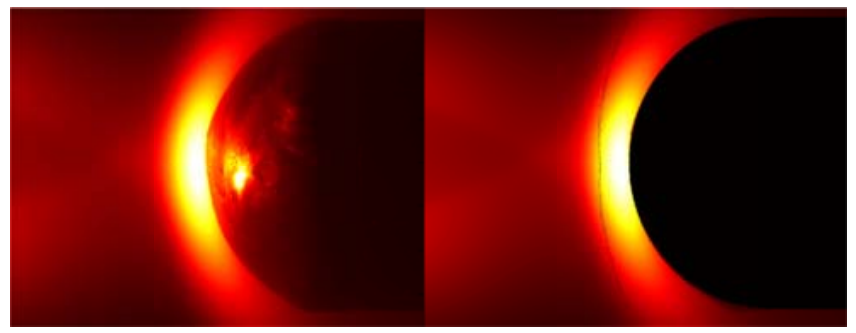

Fig. (14). Averaged picture data before and after automatic analysis.

Strictly speaking, the line intensity analysis to detect the shock position is only valid for the center line. To give an overall impression of the shock position it is nevertheless sufficient. Respectively, to define the shock position, only the center line intensity profile was analyzed.

The shock distance was referenced with respect to the probe diameter. To compare the data recorded during the magnetic field variation, a similar approach as for the temperature has been used: 
$\Delta \delta_{\text {norm }}=\frac{\delta-\delta_{0}}{\delta_{0}}$

Here, $\delta$ stands for the shock distance whereas the index 0 denotes the shock distance in the case without magnetic field present.

In earlier research the photographs have all been Abel inverted before the analysis. Due to the fact that the display detail of the pictures has been increased, the originally used Abel inversion procedure leads to high irregularities along the center line which renders the shock distance analysis impossible. On the other hand, looking at older data, the shock distance is not significantly affected by using the Abel inversion correction. Thus, within this work, Abel inversion was not applied.

The first focus was to find out about the analysis procedure and to what extend the color specific intensity distribution chosen has any influence on the bow shock position. Also, Kranc et al. and Gülhan et al. observed both a shift of the main plasma color towards red as soon as they applied the magnetic field [6, 22]. However, visually this effect was not observed during the tests at IRS. The spectral analyses in reference [23] indicate an overall increase of the ion radiation. However, the magnetic flux in our case is weaker and the data of Gülhan are apparently integrated (this is not really known from the above mentioned reference but implied).

Thus, the red center line intensity profile was also examined with respect to any possible increases due to the magnetic field present. This examination is given in Fig. (15). As can be observed, the blue intensity is about five times higher than the red one. Both intensity profiles show the same intensity reduction looking at the inflow area. Focusing on the bow shock distance, no difference was found.

As for the MHD impact on the temperature, the same variables were subject to research with respect to the bow shock distance analysis. The impact of the isolation towards the vacuum chamber as well as the coating of the probe head on the shock distance was examined.

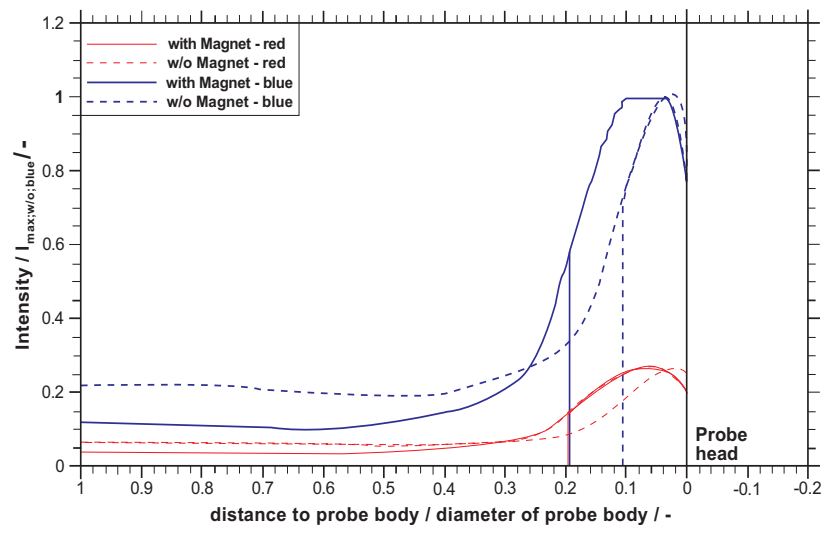

Fig. (15). Blue and red center line intensity profile in comparison.

Fig. (16) depicts the coated case. Here, the shock distance as observed in Fig. (15) is not influenced by the isolation. The rather small difference can be assigned to the measurement error. The maximum intensities of the case with and without magnet do not differ significantly. Nevertheless, by isolating the probe for the non magnetic case, the emitted light is stronger than in the grounded case.

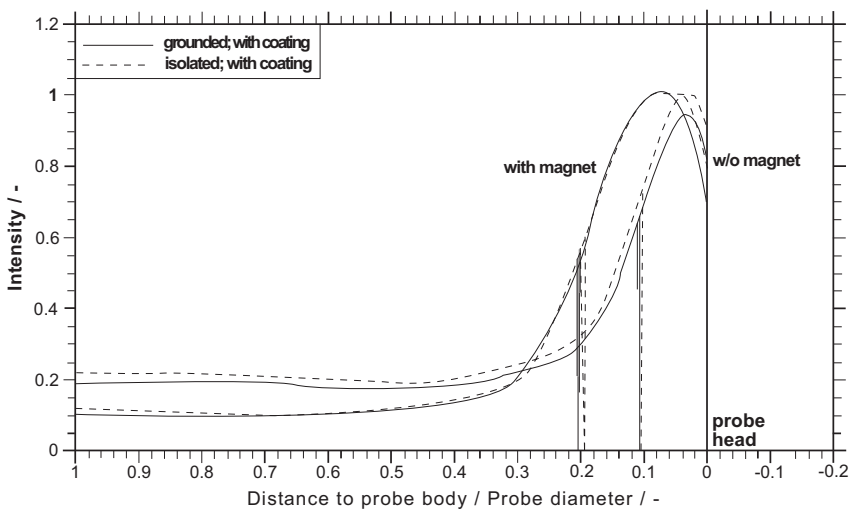

Fig. (16). Shock distance analysis for the enamel coated probe head.

Looking at Fig. (17) and the bare probe head, the shock distance is equal independent from the isolating procedure. This goes also for the intensities. Interesting is the fact that the intensity for the case without magnet is much lower compared to the one in Fig. (16) for the same case.

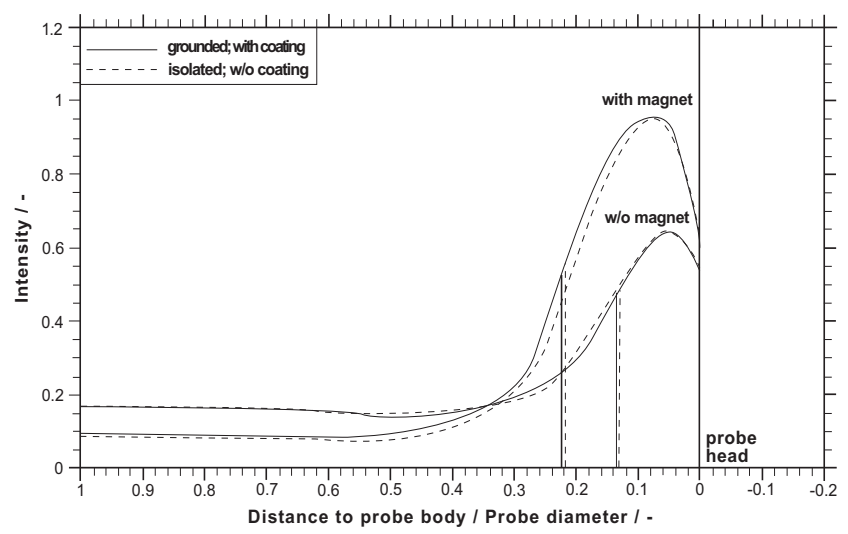

Fig. (17). Shock distance analysis for the uncoated probe head.

Comparing both cases, the isolating procedure increases the intensity of the emitted light. The bow shock is not affected by the procedure. The coating on the probe head definitely produced an effect. Also, it is very interesting, that even though no coating was used, the shock distance increased due to the magnet by the same factor. This finding stands in direct contrast to the proclamation of Otsu/Katsrayama based on their numerical approach to this type of MHD influence [13].

The observant reader will note that for the uncoated probe head, the shock distances are definitively higher. The reason is that the magnet in this case is closer to the surface of the probe. In order to apply the coating, a thin layer of copper needed to be added to the surface via electroplating. This was necessary because the enamel coating would not bond to the Kovar material [24]. Thereafter, the enamel coating could be applied which lead to an overall increase of the distance of the magnet towards to probe surface of about $0.5 \mathrm{~mm}$. Thus, field density at the probe tip was measured to be $0.339 \mathrm{~T}$ in the uncoated case. The increased distance without magnet can also be explained. The coating is not 
uniform in thickness due to the process through which the enamel coating is applied. It gets thicker towards the probe which leads to errors within the definition of the probe template being blacked out by the analysis program.

The isolated probe together with the coated probe head proved to have the strongest impact on the flow, especially with respect to the intensity. This was also one reason to choose this setup as reference setup for the magnetic field variation. The result of this variation is shown in Fig. (18).

Just as for the temperatures, the shock distance increases towards almost a factor of 2 compared to the original value. For experimental configurations with more than 3 magnets, no definite additional increase can be observed most likely due to the extent of the measurement error of about 5 Pixel, which corresponds to $0.4 \mathrm{~mm}$.

Also, a constant increase of the intensity was recorded. In contrast to the temperature and the shock distance, this value does not seem to converge towards a certain maximum value. Due to the fact that the camera is not intensity calibrated, this information still lacks the proper foundation. Thus, for future measurement campaigns, intensity calibrating the camera needs to be evaluated and possibly realized. Beyond this emission spectroscopic measurements have been performed to assess the boundary layer situation with and without magnet. This has been done in reference [24] and - on purpose- is not included in this paper as it goes far beyond the typical size of papers as such.

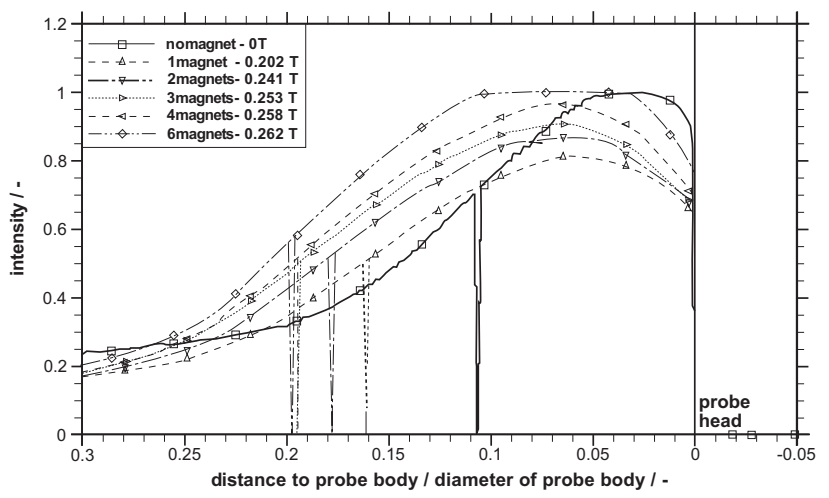

Fig. (18). Center line intensity profiles for magnetic field variation.

\subsection{Numerical Results (SAMSA)}

SAMSA [11] was used to simulate this experiment and was found capable predicting an MHD influence.

The following Figs. (19, 20) illustrate the local distribution of the electrical conductivity and the resulting Stuart numbers as calculated for the same input parameters as for the experiment.

Overall, simulating the RD5 argon flow, SAMSAs predictions with respect to the ionization degree, the conductivity and the temperatures are within the expectations However, currently the simulated Mach numbers are still too high. Thus, SAMSA is able to predict a possible MHD Effect within the flow, but it is not yet able to numerically duplicate the RD5 flow. However, the overall analysis e.g. of the Stuart number distribution as e.g. shown in Fig. (20) reproduces the position of the Hall current zone as well (the area with the local maximum of 22 for the Stuart number). A coarse comparison with the spectral data analysed in reference [24] confirms that the radial distribution of ion line intensities that have an intensity maximum around $12 \mathrm{~mm}$ from the plasma jet centre measured at an axial distance of 2 $\mathrm{mm}$ from the probe surface approves either the Hall current or the zone around the local maximum that can be seen in Fig. (20) at $x=0,268 \mathrm{~m}$ ! This in turn is the first experimental proof of the three-dimensional constellation of a MHD configuration as such and simultaneously confirms why some researchers detect temperature increases in the shoulder zones of their probes.

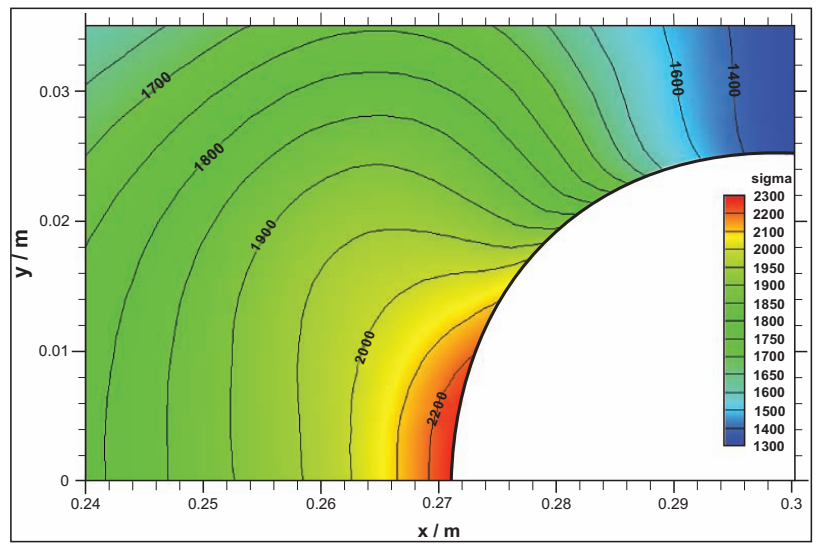

Fig. (19). Distribution of electrical conductivity by SAMSA.

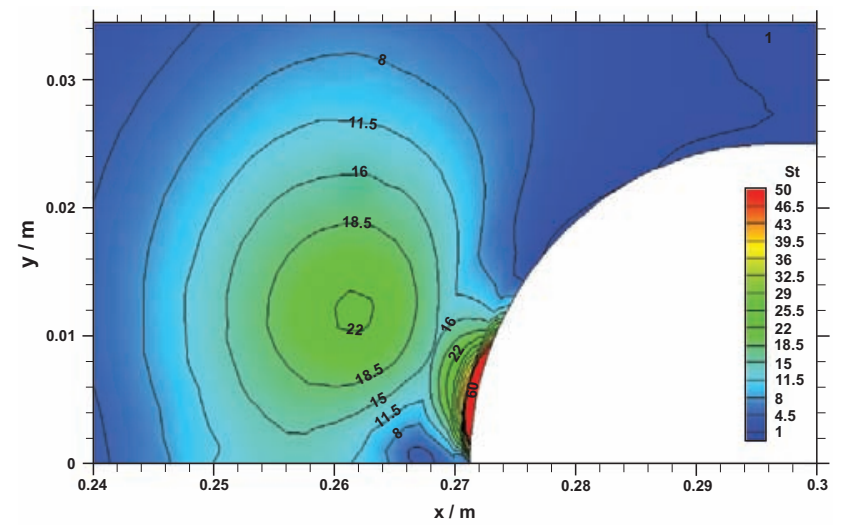

Fig. (20). Distribution of local Stuart numbers by SAMSA.

\subsubsection{Comparison of Results and Discussion}

First experiments by Kranc in the 1970s also concentrated on the magnetic field - bow shock interaction [22]. Within Fig. (21) the center line intensity profiles recorded by Kranc are compared to this work. All profiles have been normalized using the respective maximum intensity in the case without magnet as reference. As can be observed, the inflow characteristics are basically the same. The intensity profile of the magnet case is always lower than without magnet. Also, the order of magnitude of the bow shock displacement seems to be similar.

Kranc also plotted his measurement data for $\Delta \delta_{\text {norm }}$ against the applied magnetic field. Doing the same for the acquired data of this work, one gets the result displayed in Fig. (22).

Strictly speaking, this diagram is not completely valid. For one, the determination method of the bow shock distance is not the same. Also, within his research Kranc used a coil 
to generate the magnetic field and not a permanent magnet as was used within the current measurement campaign.

Nevertheless, it becomes obvious, that the measurements of the present work were achieved using a plasma condition with much higher ionization degree and electrical conductivity. Flux densities $<0.2 \mathrm{~T}$ would be interesting to fill the large gap in the test series. These can be obtained using a spacer in front of the magnets which will be part of future investigations. Also another test series at approximately half the ionization degree might lead to interesting conclusions.

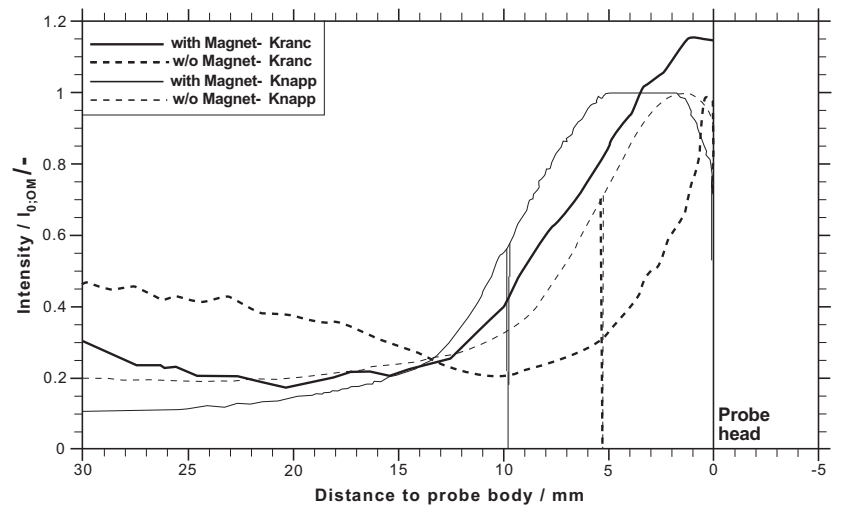

Fig. (21). Comparison of center line intensity profiles.

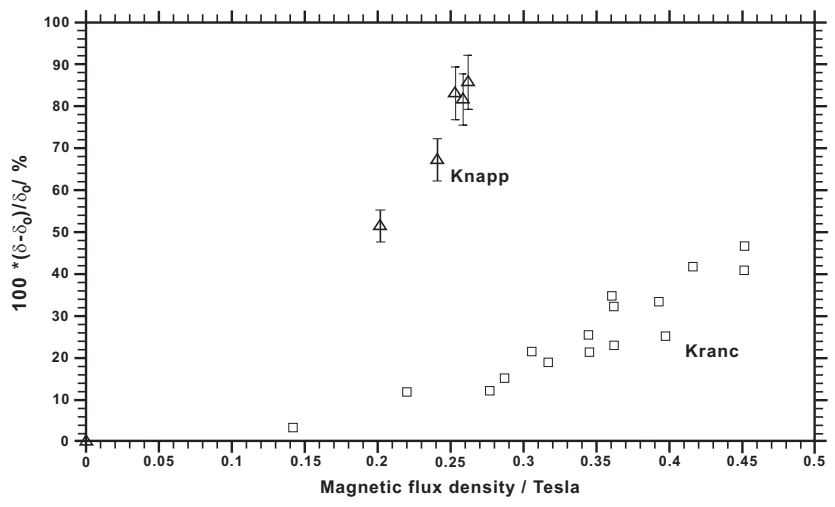

Fig. (22). Comparison of shock distance measurements.

Looking at the Hall parameter effect as described e.g. by Otsu et al. [13]

$\beta=\frac{\sigma B}{N_{e} e}=\beta_{0} B$

The proclaimed based on his numerical approach that the MHD effect of shifting the shock away from the body is negated for $\beta_{0}$ values larger than 20 , if the probe body is electrically conducting, the results of this work clearly state otherwise. Using the data from the characterization of the plasma flow, $\beta_{0}$ ranges around 470 within the applied plasma condition. Thus his numerical approach can not be applied to this PWK experiment, which might be caused by some of his simplifications e.g. the constant conductivity value for the whole calculating area. The conductivity of the copper is still four orders of magnitude higher than the one of the plasma. Thus, it is unlikely that this factor is relevant for this discrepancy.
Gülhan et al. measured in his experiment a heat flux mitigation of $46 \%$ for his respective sphere shaped model. Though he did not publish his boundary conditions detailed enough to allow a comparison or an overall assessment of these results adequately, the flux density of his setup might range within the one of Kranc. Also, the electrical conductivity value appears to be much smaller than the one within this work using the electron temperature end electron density as a basis for estimation. Given the result from Gülhan et al., the temperature reduction in our case should be more significant, seeing the high conductivity of the test Argon condition used in this investigation. In addition, he did not observe any bow shock change whatsoever, which, within this work, has been observed as a rather significant effect.

In accordance with his findings, the MHD setup presented here has similar drawbacks with respect to the inflow condition. Strictly speaking, using the magnet does not only influence the boundary layer, but also the free stream, thus changing the condition to an as yet not assessed degree. Therefore, the question is still valid, if preionized flows are suitable for these examinations.

In accordance to Fertig et al. but also to Gülhan et al., it is not valid to correlate the shock distance to the amount of heat flux reaching the probe surface $[18,6]$. Such a correlation is specific for each non equilibrium plasma condition and can therefore not be compared in between the different set-ups. The significant differences of the measurements in [18] and the ones of this paper might be an indication for this theory.

Finally, a close look on the probe head design shows that the measured temperatures within this research campaign are only taken at the same reference diameter. Looking at the MHD interaction in more detail, one finding is that it can redistribute the energy inside the shock layer. This again is confirmed by the radial spectral distributions as discussed in reference [24] and the numerical simulations shown here, see again e.g. Fig. (20). Thus, it remains still unclear, if reducing the temperature at the designated positions is equal to an overall heat flux reduction onto the whole probe head. While reducing the heat flux onto the stagnation point area, the one at a certain radial distance might very well be increased by the MHD impact. These effects are not yet fully understood and more, thorough research is necessary in order to seize the full potential of such a system.

The numerical results show that the SAMSA code is more than able to handle the MHD interaction around the probe body.

To compare the results directly is quite difficult due to the fact that e.g. the electrical conductivity can not be measured directly. The electron temperature as well as the number density of the electrons can be measured using electrostatic probe measurements. Nevertheless, there are matching results and the overall MHD effects using this plasma condition are significant as can be seen by the effect on the temperature and radiation. In addition, a comparison with the radial profiles taken with emission spectroscopy show that the three-dimensional behavior of the overall effect e.g. in terms of Hall current zone can be traced. 
However, the data still have to be investigated more in detail as the measurements may be influenced by the shock itself.

Overall the results are promising and it is expected, that using a coating material for the experiment as well as a respective boundary condition for the numeric simulation will yield more definite results.

\section{CONCLUSION}

Tests have been performed using argon as working gas at different plasma conditions. The temperature of the probe head has been measured and pictures have been analyzed in order to get first results concerning the heat flux onto the probe body as well as changes in the bow shock geometry.

The same input variables of this experiment were used to do a SAMSA numerical simulation.

In a next step the plasma condition has to be assessed in detail. However, as mentioned in the paper, both intrusive probe measurements such as enthalpy and Langmuir probes and non-intrusive methods such as emission spectroscopy have already been performed successfully and reported in other papers. The data presented here and the plasma diagnostic assessment signify, therefore, the first reference plasma condition for later numerical analysis.

(1) The temperature measurements did not yield any definite results. The measurements of one sensor show an increase of the temperature using the magnet. This effect has to be analyzed further.

(2) The bow shock analysis yielded a slight increase in bow shock distance, though more experiments are necessary to get adequately ascertained quantitative results.

(3) Additional experiments using an isolating coating material on the probe head are necessary in order to evaluate the influence of the Hall Effect on the temperatures, as well as on the bow shock geometry.

(4) The numerical analysis shows, that the SAMSA code is efficient in handling this MHD problem and that key characteristics of the plasma flow show a good consistency.

(5) The comparison of the experimental and the numerical results show both that for the plasma condition investigated here the MHD interaction is fairly low.

(6) Further numerical analyses using adjusted boundary condition are necessary to get better results.

(7) Qualitative comparisons with radial profiles of spectral intensities for Argon ions show an agreement with the overall result in Fig. (20), an evidence which takes the three-dimensional effects of the MHD effect into account by both numerical analysis and experiment.

\section{ACKNOWLEDGEMENTS}

The authors thank all students and especially B. Wollenhaupt for their contribution to this work.
This work was supported by the German Research Foundation (DFG) within the funding programme Open Access Publishing.

\section{CONFLICT OF INTEREST}

Declared none.

\section{REFERENCES}

[1] Herdrich G, Auweter KM, Fertig M, Nawaz A, Petkow D. MHD flow control for plasma technology applications. Vacuum 2006; 80: 1167-73.

[2] Knapp A, Auweter KM, Fertig M, Herdrich G, Löhle S. Investigation of a magnetic probe to influence weakly ionized plasma. Proceedings of the $37^{\text {th }}$ AIAA Plasmadynamics and Lasers Conference 2006 June, San Francisco, USA.

[3] Knapp A, Fertig M, Haag D, Herdrich G, Auweter KM. Investigation of the interaction between a magnetic probe body and argon plasma. Proceedings of the $38^{\text {th }}$ AIAA Plasmadynamics and Lasers Conference; 2007 June, Miami, Florida, USA.

[4] Gaisbauer U, Knauss H, Wagner S, et al. Flow simulation and problems in ground test facilities. In: Jacob D, Sachs G, Wagner S Eds. Basic research and technologies for two-stage-to-orbit vehicles. Final Report on Collaborative Research Centres 253, 255 and 259. USA: Wiley-VCH Verlag GmbH \& Co. KgaA 2005; pp. 165-99.

[5] Borghi CA, Carraro MR, Christofolini A. Analysis of magnetoplasmadynamic interaction in the boundary layer of a hypersonic wedge. J Spacecr Rockets 2005; 42(1): 45-50.

[6] Gülhan A, Koch U, Siebe F, et al. Experimental verification of heat flux mitigation by electromagnetic fields in partially-ionized-argon flows. J Spacecr Rockets 2009; 46(2): 274-83.

[7] Katsurayama H, Kawamura M, Matsuda A, Abe T. Particle simulation of electromagnetic control of a weakly ionized flow past a blunt body. Proceedings of the $45^{\text {th }}$ AIAA Aerospace Sciences Meeting and Exhibit 2007; 8-11 January, Reno, Nevada, USA.

[8] Knapp A. Experimentelle untersuchung von magnetohydrodynamischen einflüssen auf plasmaströmungen. Doctoral thesis (in German). Germany: Institute of Space Systems, University of Stuttgart, 2012.

[9] Marvin JG, Pope RB. Laminar convective heating and ablation in the mars atmosphere. AIAA J 1967; 5(2): 240-8.

[10] Herdrich G, Fertig M, Löhle S. Experimental simulation of high enthalpy planetary entries. Open Plasma Phys J 2009; 2(15):15064.

[11] Haag D, Auweter KM, Fertig M, Herdrich G. Numerical simulations and accompanying experimental investigations of magnetoplasmadynamic thrusters with coaxial applied magnetic field. Trans Space Technol Japan 2009; 7(ists26): pp.Tb_19-Tb_28.

[12] Knapp A, Herdrich G, Auweter KM. Magnetic influence on argon plasma flow using permanent magnets. J IAPS 2007; 15(2): 14955 .

[13] Otsu H, Abe T, Konigorski D. Influence of the hall effect on the electrodynamic heat shield system for reentry vehicles. Proceedings of the $36^{\text {th }}$ AIAA Plasmadynamics and Lasers Conference, 2005 June 6-9; Toronto, Ontario Canada.

[14] Femm.info [Home page on internet] author: Meeker D. Available from: www.femm.info [Updated: $24^{\text {th }}$ June 2011 ; cited: $23^{\text {rd }}$ May 2012]

[15] Ono N, Knapp A, Haag D, Fertig M, Herdrich G. Analysis of argon plasma jet around blunt and cone probe body. J IAPS 2008; 16(1): $1-6$.

[16] Kodak.com [Home page on internet]. Available at: www.kodak com Kodak KAF5101CE CCD Color Image Sensor 1.0. [updated: unknown; cited: $23^{\text {rd }}$ June 2003].

[17] Tanifuji T, Matsuda A, Wasai K, et al. Experimental investigation of electrodynamic heat shield effect in a reentry plasma. Proceedings of the $39^{\text {th }}$ Plasmadynamics and Laser Conference 2008, 23-26 June, Seattle, Washington, USA.

[18] Fertig M, Winter M, Herdrich G, Röser H-P. Modelling of high speed earth entry. Study Note for ESA. Report No.: P2.2. Stuttgart, Germany: Institute of Space Systems 2008.

[19] Knapp A, Löhle S, Herdrich G, Auweter KM. Investigation of a magnetic heat flux probe in argon plasma flow. Proceedings of the 
$40^{\text {th }}$ AIAA Plasmadynamics and Lasers Conference AIAA-20093905; 2009 June 22-25; San Antonio, Texas, USA.

[20] Löhle S, Knapp A, Herdrich G, Auweter KM. Local enthalpy estimation in plasma flows using an improved mass injection probe. Proceedings of the $16^{\text {th }}$ AIAA/DLR/DGLR International Space Planes and Hypersonic Systems and Technologies Conference AIAA-2009-7301; 2009 October 19-22; Bremen, Germany.

[21] Heiermann J. Ein finite-volumen-verfahren zur lösung magnetoplasmadynamischer erhaltungsgleichungen. Doctoral thesis (in German). Germany: Institute of Space System, Universität Stuttgart 2002.
[22] Kranc S, Yuen MC, Cambel AB. Experimental investigation of magnetoaerodynamic flow around blunt bodies. NASA Contractor Report, August 1969.

[23] Wernitz R, Knapp A, Eichhorn C, et al. Emission spectroscopic investigation of the radial distribution of ArI and ArII in argon plasma flows under the influence of a magnetic field. Proceedings of the $42^{\text {nd }}$ AIAA Plasmaphysics and Lasers Conference AIAA 2011-3455; 2011 June 27-30; Honolulu, Hawaii, USA.

[24] Knapp A, Haag D, Ono N, Fertig M, Herdrich G, Auweter KM. Experimental and numerical analysis of the impact of a strong permanent magnet on argon plasma flow. Proceedings of the $40^{\text {th }}$ AIAA Plasmadynamics and Lasers Conference AIAA-2009-3904; 2009 June 22-25; San Antonio, Texas, USA.

(C) Knapp et al.; Licensee Bentham Open.

This is an open access article licensed under the terms of the Creative Commons Attribution Non-Commercial License (http://creativecommons.org/licenses/by-nc/3.0/) which permits unrestricted, non-commercial use, distribution and reproduction in any medium, provided the work is properly cited. 